\title{
Utilización de Simulación de Eventos Discretos para analizar una propuesta de mejora en los costos de almacenamiento
}

\section{Using Discrete Event Simulation to analyze a proposal of improve storage costs}

DOI: https://doi.org/10.17981/bilo.3.1.2021.01

Fecha de Recepción: 11/01/2021. Fecha de Publicación: 15/03/2021

\author{
Thalía Ahumada Morales \\ tahumada2@cuc.edu.co \\ Hernando Casalins-Barrios \\ hcasalin1@cuc.edu.co \\ Yemsy Mercado-Guardiola \\ ymercado19@cuc.edu.co \\ Enymar Ruiz-Comas \\ eruiz10@cuc.edu.co
}

Alexander Troncoso-Palacio

atroncos1@cuc.edu.co

Departamento de Productividad e Innovación

Universidad de la Costa. Barranquilla, (Colombia)

\begin{abstract}
Resumen. El almacenamiento prolongado en cualquier empresa es un problema, porque aumenta los costos de uso del almacén, además, la mercancía que se almacena por un tiempo prolongado puede resultar dañada, razón por la cual se planea este estudio. Donde se muestra la implementación de un modelo de simulación de eventos discretos para analizar unas posibles mejoras en los costos de almacenamiento en una compañía de tecnología móvil, con el objetivo de proponer mejoras que reduzcan los costos actuales del sistema. Para ello, se seleccionaron los 3 productos con mayor rotación. Se utilizó el software Arena ${ }^{\circledR}$ para modelar el sistema a partir de datos históricos de las demandas y los pedidos realizados en los períodos anteriores. Luego de aplicar el modelo se encontró que una buena estrategia para reducir costos es modificar la cantidad solicitada, más específicamente aumentar la de los productos 1 y 2 , y reducir la del producto 3 . En los resultados se pudo dar cuenta de que la mayoría de los desabastecimientos en el inventario son de los dos primeros productos. Por tanto, se propusieron diferentes propuestas para cada uno de los productos; Por tanto, se concluye que la propuesta 1 es la más recomendada de utilizar, ya que no es muy invasiva y no afecta la relación de clientes y proveedores.

Palabras clave: Simulación de Eventos Discretos, Inventario de mercancía, Costos de almacenamiento, Productos faltantes.
\end{abstract}

Abstract. Prolonged storage in any company is a problem, because it increases the costs of using the warehouse, in addition, merchandise that is stored for a long time can be damaged, which why this study it is planned. Where is shows the implementation of a simulation models in the storage process of a mobile technology marketing 


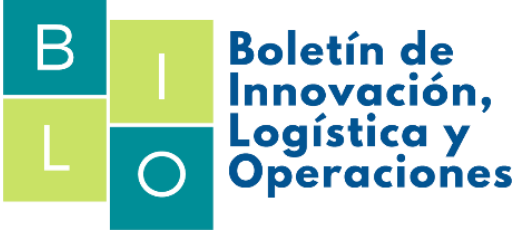

company, with the aim of proposing improvements that reduce the current costs of the system. This, 3 products with the highest turnover were selected. It used Arena ${ }^{\circledR}$ software to model the system from historical data of the demands and the orders made in the previous periods. After applying the model, it was found that a good strategy to reduce costs is to modify the quantity request, more specifically to increase that of products 1 and 2 , and reduce that of product 3 . The results showed that the biggest inventory stock out are of the first two products. Therefore, different alternatives were proposed for each one of the products; Therefore, it is concluded that proposal 1 is the most recommended to use, due since it is not very invasive and does not affect the relationship of customers and suppliers.

Keywords: Discrete Event Simulation, Merchandise inventory, Storage costs, Missing products.

\section{Introducción}

Según estudios realizados por diferentes empresas distribuidoras, incluyendo también las de tecnología pudimos observar que la mayor dificultad en temas de inventario es saber cuándo hacer un pedido y que cantidad pedir, tomar una decisión acertada le hará ganar o perder dinero sustancialmente. Por ello un buen sistema de inventario evitará el agotamiento de existencias y permitirá un amplio margen de tiempo para reponer la mercancía faltante y dará una idea del valor atado a las mismas en cualquier momento dado. Para muchas empresas el inventario físico es su mayor gasto, la adecuada gestión de este gasto podría ser significativa para un resultado exitoso. Desde la hipótesis que dice que, para una buena gestión en los inventarios, es necesaria una política de inventario, cual ayudaría a mejorar los flujos de las mercancías en existencias. Los sistemas de control de inventario toman una medida precisa de los activos actuales, inventario en reserva, y proporcionan una mejor idea sobre la situación financiera. La política de inventario también es esencial primordial para el adecuado equilibrio de los diferentes productos almacenados, un stock insuficiente puede provocar una parada de la producción, además de que pueden crear un impacto negativo en los clientes. Una sólida política de inventario asegurará que una empresa tenga suficientes materias primas para llevar a cabo sin problemas la fabricación y el suministro de los productos terminados. La política de inventario también desempeña un papel crucial para evitar la sobreacumulación. Diversas técnicas de gestión de inventario garantizan que los productos no permanezcan inactivos en los almacenes. Además, algunas empresas pueden integrar la metodología de eliminación para reemplazar los productos excedentes por medio de desmontajes y remodelaciones. La falta de una política de inventario puede causar el cierre completo de un negocio. Para evitarlo, las empresas suelen implementar estrategias que integran al proveedor de la materia prima, a los departamentos de fabricación de su empresa, y a las empresas de estructura logística o de transporte para minimizar la acumulación o el desperdicio de los productos terminados. En este trabajo se busca implementar modelos de inventarios en la empresa de tecnología para establecer una nueva política de inventarios que se desempeñe mejor que la política actual, para ello se realizará una toma de datos de costos de inventario, demanda y pedidos realizados en el anterior periodo, con el fin de simular diferentes políticas de inventario y encontrar la más adecuada que reduzca los costos de este proceso en el futuro. Muchos autores han estudiado esta problemática y en la divulgación de sus informes se pueden encontrar algunos como los mencionados a continuación.

\section{Revisión Bibliográfica}

En las diferentes bases de datos de divulgación de investigaciones, existen muchos estudios basados en la aplicación de modelos de inventarios, como por ejemplo: En la investigación realizada por [1], donde se evidencia la interacción entre la atención al cliente y el inventario, en una compañía comercializadora de productos, donde se utilizaron herramientas estadísticas como la prueba de hipótesis para determinar la relación entre las dos variables, dando como resultado una correlación positiva. Por lo anterior se concluyó que el control de inventarios impacta radicalmente el proceso de atención al cliente. También teniendo en cuenta el estudio de [2],el cual aplico el modelo EOQ para reducir el costo de inventario, en una compañía productora de cerveza en Tailandia. La empresa tenía problemas en cuanto al alto costo de los inventarios por la pobre gestión de los mismo. La causa fundamental encontrada fue la falta de una política de inventario sólida, lo que implicaba un alto costo con exceso de inventario, o tener muy poco para cumplir la demanda. También significaba pagar un alto costo de almacenamiento. Al simular el modelo el resultado indica que la empresa puede conseguir un ahorro de costes que asciende al 50\% del antiguo coste del inventario. Estudiando de igual manera el estudio de [3], donde evaluaron mediante un modelo en el software Arena las políticas de inventarios en una compañía del sector farmacéutico, lo cual dejó en evidencia un alto porcentaje en la atención al cliente. También en la investigación de [4], utilizaron la simulación para hallar la óptima cantidad de pedido y la periodicidad de la misma, y el nivel de servicio paso del $72 \%$ al $99 \%$ mejorando en un $27 \%$. Igualmente en [5], mediante simulación Monte Carlo, estudiaron el caso de una empresa de fabricación electrónica, diseñaron un modelo de simulación que imitaba el sistema de inventario actual y se utilizó para estudiar el sistema actual y las políticas alternativas de puntos de reordenación. Se experimentaron varios puntos de reordenación para determinar la política de reordenación que da como resultado 


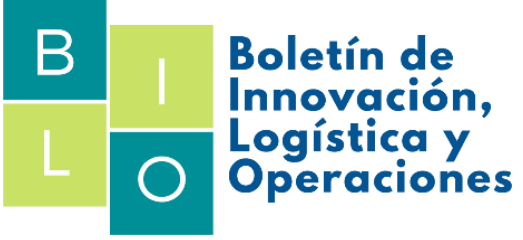

el costo total de inventario promedio más bajo por semana. Asimismo [6], se evaluó la rotación de los productos en una bodega para ubicarlos en las mejores posiciones de tal manera que se recorran menos distancias al momento ser solicitados. En la investigación de [7], se realizó una propuesta analizada mediante el software Arena, donde se evidenció que, para aumentar el nivel de servicio, se debe implementar un plan de inventario que tiende a incrementar las unidades en las líneas donde se encontró una mayor demanda y adicionalmente realizar la labor de mercadeo en recuperación de clientes perdidos por esa razón. Además en [8], Estudiaron un sistema de inventarios en una cadena de suministros con el objetivo de determinar las políticas de inventario apropiadas que pueden ayudar a una empresa a lograr ahorros de costos y la satisfacción de sus clientes en lo que se refiere a los plazos de entrega, con un enfoque de modelización por simulación para un ejemplo numérico de una cadena de suministro que incluye al fabricante y al distribuidor, se analizó un conjunto de cantidad de pedidos, punto de reordenación o período de revisión para diferentes políticas de inventario en función del costo total del inventario y la demanda perdida, el resultado fue que se ayudar a los encargados de la toma de decisiones en la compañía a lograr la mejora en el nivel de servicio. Por otro lado, en el estudio de [9], propusieron un modelo de optimización basado en la simulación del sistema de inventario multi-echelon para productos agrícolas frescos utilizando el software de simulación FlexSim y el algoritmo mejorado de optimización de enjambres de partículas, los resultados de la simulación demuestran que el modelo propuesto de optimización basado en la simulación del sistema de inventario llega a proporcional soporte en la toma de decisión en el control de inventarios, debido a que la modelización del sistema es un método eficaz para resolver problemas complejos. En la investigación de [10], los resultados identificaron el cuello de botella y en base a la simulación se obtuvo una mejora la productividad en un $27,45 \%$, lo que representa para esa compañía el procesamiento de otro elemento en una de sus áreas. Apoyándonos en el estudio de [11], donde desarrollaron y validaron un de un proceso con la identificación de los posibles factores de mejora a través de un análisis causa-efecto, seguido de la aplicación de modelos de inventarios, planteando estrategias como la identificación y rotación de productos, control de entradas y salidas de mercancías y uso de indicadores de gestión. En el estudio de [12], utilizaron la simulación en la gestión del inventario por medio del software Arena, donde plantearon las siguientes recomendaciones a la compañía: 1.) Aumentar el punto de reordenación de 2.000 unidades a 2.500 unidades, con el fin de actualizar el inventario nivelar más rápido y disminuir la probabilidad de pérdida clientes y ventas; 2) Disminuir el tamaño del lote de 9.000 unidades a 8.000 unidades; 3) Aumentar el stock objetivo de 10.000 unidades a 11.000 unidades, lo que nos mostró la mejor mejora ya que reduce el porcentaje perdido a aproximadamente el 1\%. Analizando también el estudio [13], estudiaron el sistema de inventario de las instalaciones en la Universidad Estatal del Sudeste de Missouri, el problema que se abordó fue la falta de una adecuada gestión de inventario generador de costos excesivos en el almacén de mantenimiento de las instalaciones, frecuentemente se agotan las existencias de lámparas, lo que cuesta dinero extra debido a una orden urgente hecha, luego de aplicar el modelo encontraron que el almacén de mantenimiento debería utilizar un mayor tamaño de lote y otro punto de re-orden para minimizar el costo y el stock de su inventario. De igual manera el estudio realizado por [14], quienes realizaron un análisis de las alternativas de los softwares comerciales. En [15], se analizaron las reducciones a través de un modelo considerando dos minoristas idénticos con un centro de distribución y un fabricante, la simulación del modelo fue llevada a cabo a través del software Arena para optimizar el inventario. En el estudio de [16], se modelo el sistema para conocer el comportamiento de las entradas y salidas de la mercancía en inventario. En [17], se formuló una metodología para que los estudiantes diseñaran simulaciones en la cadena de suministros. En [18], se presentó una propuesta para mejorar las exportaciones. Finalmente, con base en la investigación de [19], donde utilizaron simulación para analizar un proceso minero, llegaron a la conclusión de que deberían aumentar los elementos encargados de la distribución para mejorar la productividad. También en [20], quienes emplearon un modelo simulado por medio del software FlexSim en el proceso de almacenamiento de un almacén, con el fin de identificar los cuellos de botellas, eliminándolos para optimizar el sistema. Conforme a esta revisión se propone seguir la siguiente metodología [21].

\section{Metodología}

La metodología planteada en este proyecto es de tipo explicativa, teniendo en cuenta que dicha metodología además de describir el problema, se acerca a las causas y posibles soluciones y esta se realizará de la siguiente manera: en el primer paso se caracterizará el proceso con el fin de determinar que productos están generando mayores problemas en el inventario, y enfocar esta investigación a ellos; seguido de esto se realizará un análisis de entrada de las entidades que en este caso serán las demandas de los productos de mayor rotación; posteriormente se realiza el diseño del modelo conceptual, en el cual se detalla cómo es el comportamiento del proceso de inventario. Seguido a esto se ejecutará una recopilación de los datos necesarios para poder simular estos procesos, estableciendo el tamaño de la muestra que se debe tener para estos y hallando las respectivas distribuciones de las entidades y recursos que se necesitaran para implementar la simulación. A continuación, se definen las variables posibles que se pueden presentar en el proceso y que pueden afectar en la simulación en el software; luego se simula el modelo en el programa y se valida con los datos del proceso real y por ultimo de acuerdo con el costo 


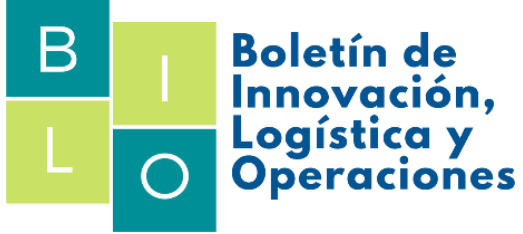

obtenido se determinará cual política se ajusta mejor para la compañía. Esta metodología se desarrolla a continuación. La secuencia se puede observar en la figura 1.

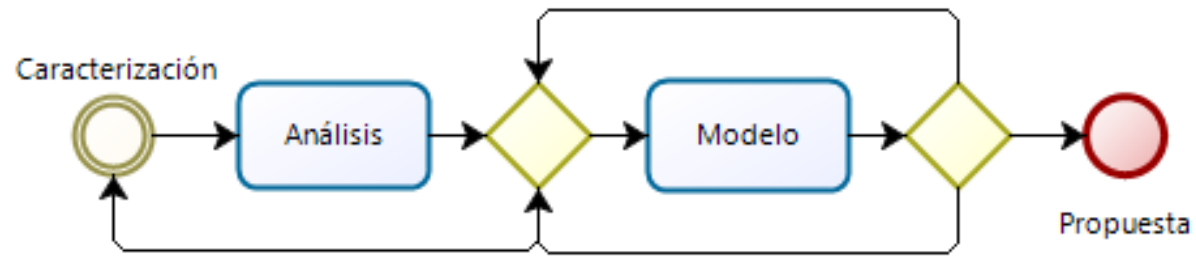

Figura 1. Metodología propuesta

\section{Desarrollo}

Aplicando la metodología propuesta se inicia con la caracterización del proceso, seguido de un análisis de entrada, sucesivo a esto se realiza el diseño del modelo conceptual, posteriormente se ejecutará el modelo de simulación, continuando con el proceso, se definen las variables posibles, después se corre el modelo antes diseñado y por último se comparas los diferentes escenarios para determinar la mejor política de inventario que se ajusta a la compañía. Se estudiarán 3 referencias las cuales son las de mayor rotación; se recolectaron datos para el análisis de entrada del modelo para cada una de las tres referencias de mayor rotación denominadas con Ref. 1, 2, 3. A estos datos se les aplico una prueba de bondad de ajuste por medio del software Statgraphics para determinar a qué tipo de distribución es semejante.

\section{A. Análisis de datos de entrada}

A continuación, se muestra el histograma de frecuencias (Figura 1) de los tiempos entre pedidos para la referencia 1, además del informe emitido por el software el cual aclara la distribución de mejor ajuste a los datos.

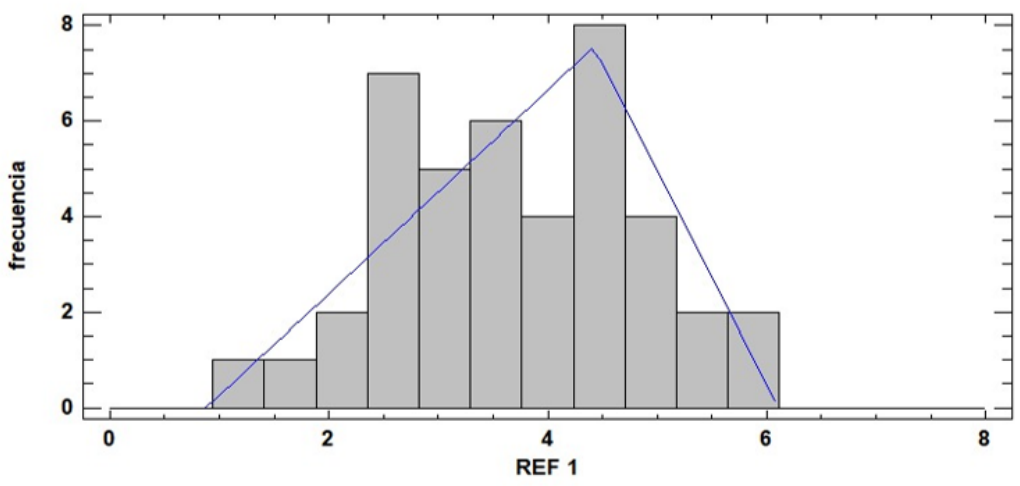

Distribución Triangular

Límite inferior. $\quad 0,877911$

Moda.

4,42124

Limite Superior. $\quad 6,11075$

Prueba K-S

Valor-P

0,795734

Figura 2. Prueba de bondad de ajuste pedidos para la referencia 1.

Teniendo en cuenta que el valor-p es mayor a la significancia 0.05 , se acepta la hipótesis que la referencia 1 proviene de una distribución triangular con un $95 \%$ de confianza. A continuación, se muestra el histograma de frecuencias (Figura 2) de los tiempos entre pedidos para la referencia 2, además del informe emitido por el software el cual aclara la distribución de mejor ajuste a los datos.

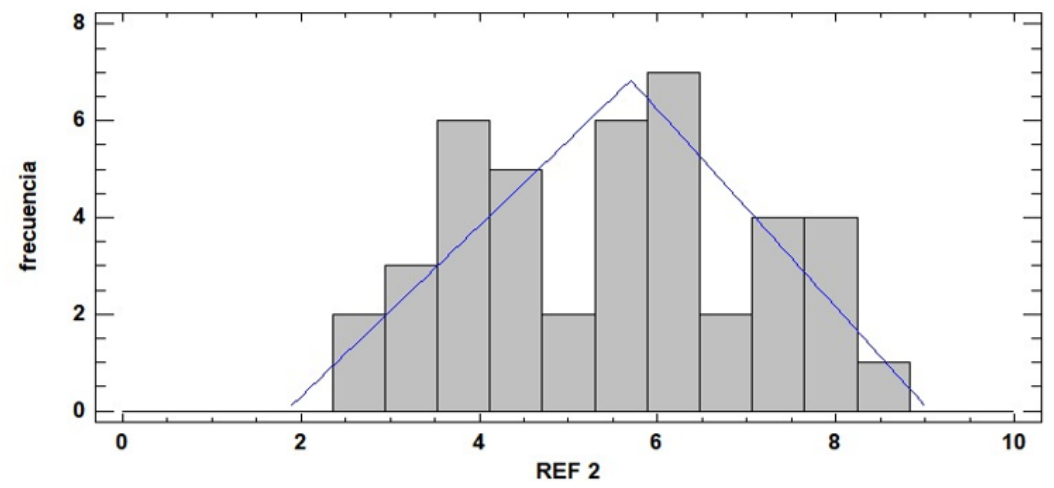

Distribución Triangular

Límite inferior. $\quad 1,82926$

Moda. $\quad 5,70367$

Limite Superior. $\quad 9,04937$

Prueba K-S

Valor-P $\quad 0,835817$

Figura 3. Prueba de bondad de ajuste pedidos para la referencia 2.

Teniendo en cuenta que el valor-p es mayor a la significancia 0.05 , se acepta la hipótesis que la referencia 2 proviene de una distribución triangular con $95 \%$ de confianza. A continuación, se muestra el histograma de 


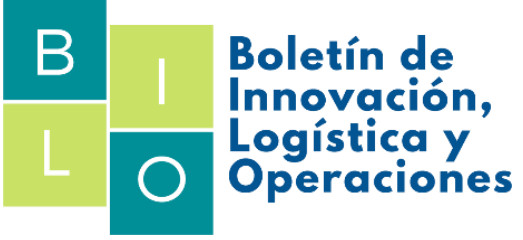

frecuencias (Figura 3) de los tiempos entre pedidos para la referencia 3, además del informe emitido por el software el cual aclara la distribución de mejor ajuste a los datos.

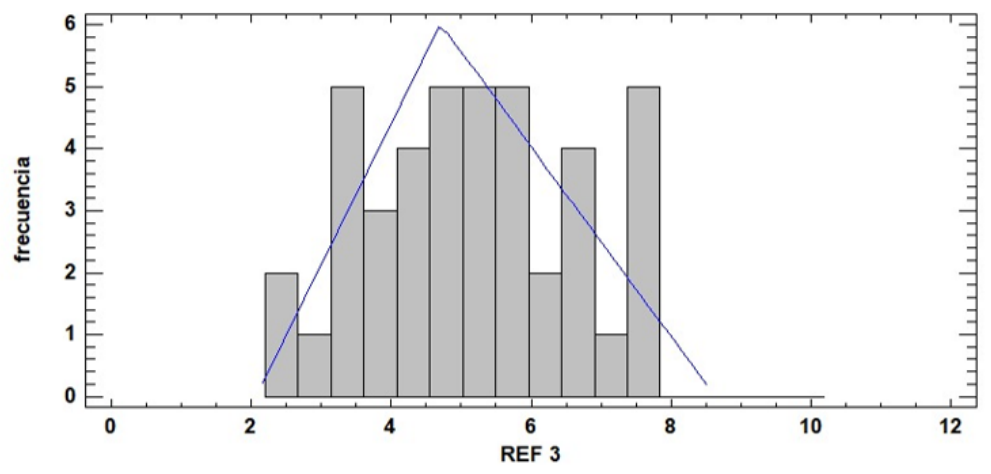

Distribución Triangular

Límite inferior. 2,06698

Moda. 4,70309

Limite Superior. $\quad 8,63505$

Prueba

$\mathrm{K}-\mathrm{S}$

Valor-P

Figura 4. Prueba de bondad de ajuste pedidos para referencia 3.

Teniendo en cuenta que el valor-p es mayor a la significancia 0.05 , se acepta la hipótesis que la referencia 3 proviene de una distribución triangular con $95 \%$ de confianza.

\section{B. Modelo descriptivo del sistema de inventario}

Se tiene que, para la llegada de los pedidos de los clientes, los cuales solicitan una cantidad de producto en función de la referencia solicitada, dado históricos y experiencia en el área comercial se obtiene que el comportamiento de los pedidos sigue una distribución de probabilidades evidenciadas en la tabla 1.

Tabla 1. Porcentaje de pedidos

\begin{tabular}{cccccc}
\hline \multirow{2}{*}{ Referencia } & \multicolumn{5}{c}{ Cantidad } \\
& 1 & 4 & 6 & 8 & 10 \\
\hline 1 & $1 \%$ & $10 \%$ & $20 \%$ & $25 \%$ & $44 \%$ \\
2 & $20 \%$ & $25 \%$ & $30 \%$ & $15 \%$ & $10 \%$ \\
3 & $85 \%$ & $10 \%$ & $4 \%$ & $1 \%$ & $0 \%$ \\
\hline
\end{tabular}

Luego de solicitado el pedido por el cliente se verifica si existe inventario para cumplir la orden, en caso de que no se tenga se genera un faltante por el valor de la cantidad de pedido solicitado, de otro modo se retira de inventario la cantidad solicitada y se sale del sistema. Para el subsistema de abastecimiento se parte de una orden de compra o pedido solicitado por la empresa a su proveedor, en donde se pide una cantidad específica que actualmente es de 50 unidades de la referencia 1, 20 de la 2 y 10 de la 3 al mes. Se puede evidenciar la secuencia a seguir dependiendo del inventario en la figura 5

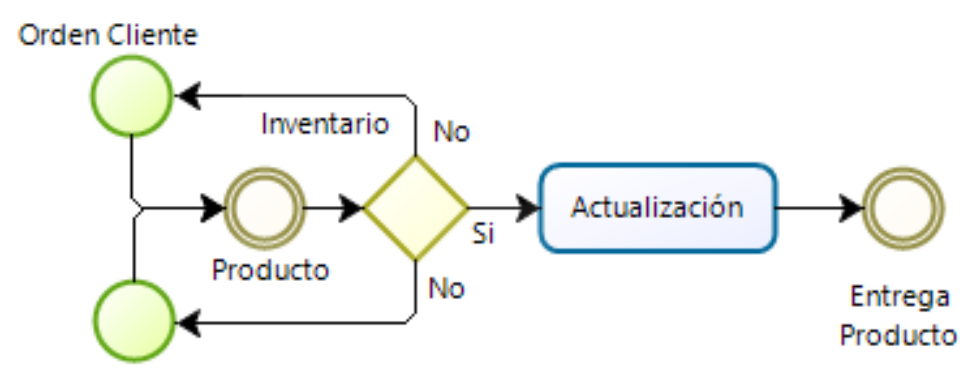

Suministro

Figura 5. Secuencia de solicitud de equipo.

Luego de esto el proveedor procesa la orden en un lead time que sigue una distribución uniforme de 20 a 35 días. Llegada la mercancía a bodega se registra el aumento del inventario y se sale de este sistema. A continuación, se enuncian todos los elementos del modelo con sus respectivas características. Cabe resaltar que a menos que se diga lo contrario todas las unidades estarán definidas en días. Para el modelo se plantearon 6 entidades referentes a lo solicitado por los clientes y los proveedores, la Tabla 2 resume las características de estas entidades. 
Tabla 2. Características

\begin{tabular}{cc}
\hline Entidad & Distribución \\
\hline Pedido 1 & Triangular \\
& $(0.88,4.42,6.11)$ \\
Pedido 2 & Triangular \\
& $(1.83,5.7,9.05)$ \\
Pedido 3 & Triangular \\
Producto 1 & $(2.07,4.7,8.63)$ \\
Producto 2 & COST (30) \\
Producto 3 & COST (30) \\
\hline
\end{tabular}

Se emplearon 3 variables que representan la cantidad de inventario a tiempo real de cada uno de los productos, con esto es posible recalcular la cantidad de inventarios luego de realizado un pedido o de reabastecerse de producto la bodega. Fueron usados 3 procesos para definir el modelo referente al lead time de cada proveedor que como se mencionó anteriormente sigue una distribución UNIF $(20,35)$ independientemente del tipo de producto. El modelo tiene 6 salidas que emulan la salida de cada uno de los subsistemas para cada producto. Para el modelado del sistema se tienen en cuenta los siguientes supuestos: cada orden emitida por los clientes es única, es decir, se tendrá una orden por cada tipo de producto que el cliente requiera en caso de presentarse el mismo día. Cada orden de compra de producto a los proveedores es única, es decir, en caso de solicitar más de una referencia en la misma fecha se tomará esta como una nueva orden. No se incluyen los tiempos de almacenamiento o retiro del producto en bodega ya que es despreciable con respecto al tiempo entre llegada de pedidos y de reabastecimiento del producto. Se supone que se tiene una capacidad de inventario en la bodega infinito dado que los componentes no ocupan mucho espacio y además tienen lead times muy largos, por lo que es inconcebible que se llegue a ocupar todo el espacio de la bodega.

\section{Modelo en el software arena}

Luego de una corrida de simulación para un año se obtuvieron faltantes de 21, 23 y 3 para las referencias 1,2 y 3 , respectivamente. Además, se presenta en la Figura 5 el comportamiento del nivel de inventarios de cada producto a lo largo de una simulación de 1 corrida de 1 año completo. El modelo se muestra en la Figura 6.
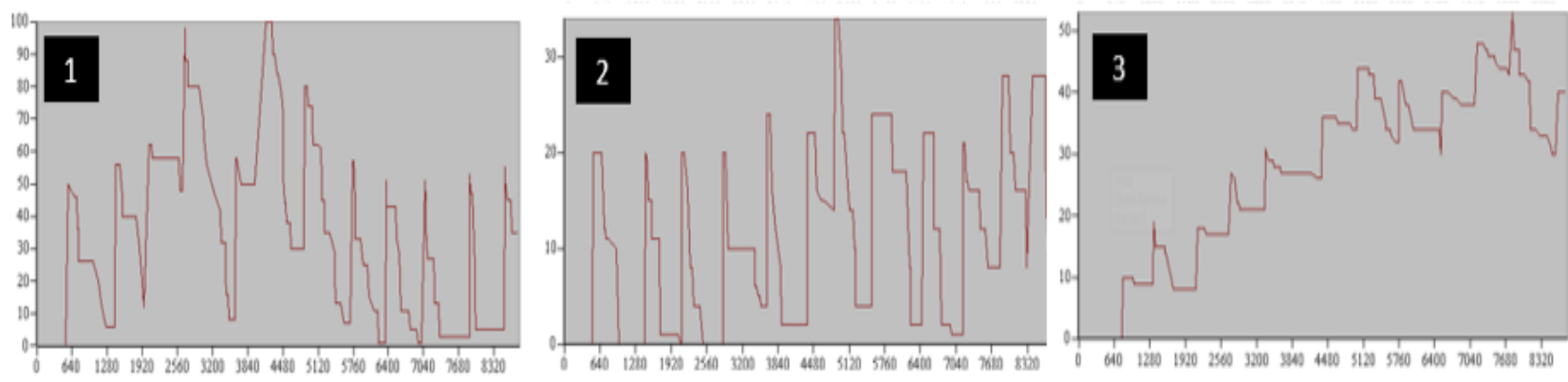

Figura 6. Comportamiento del nivel de inventario en el prototipo. 

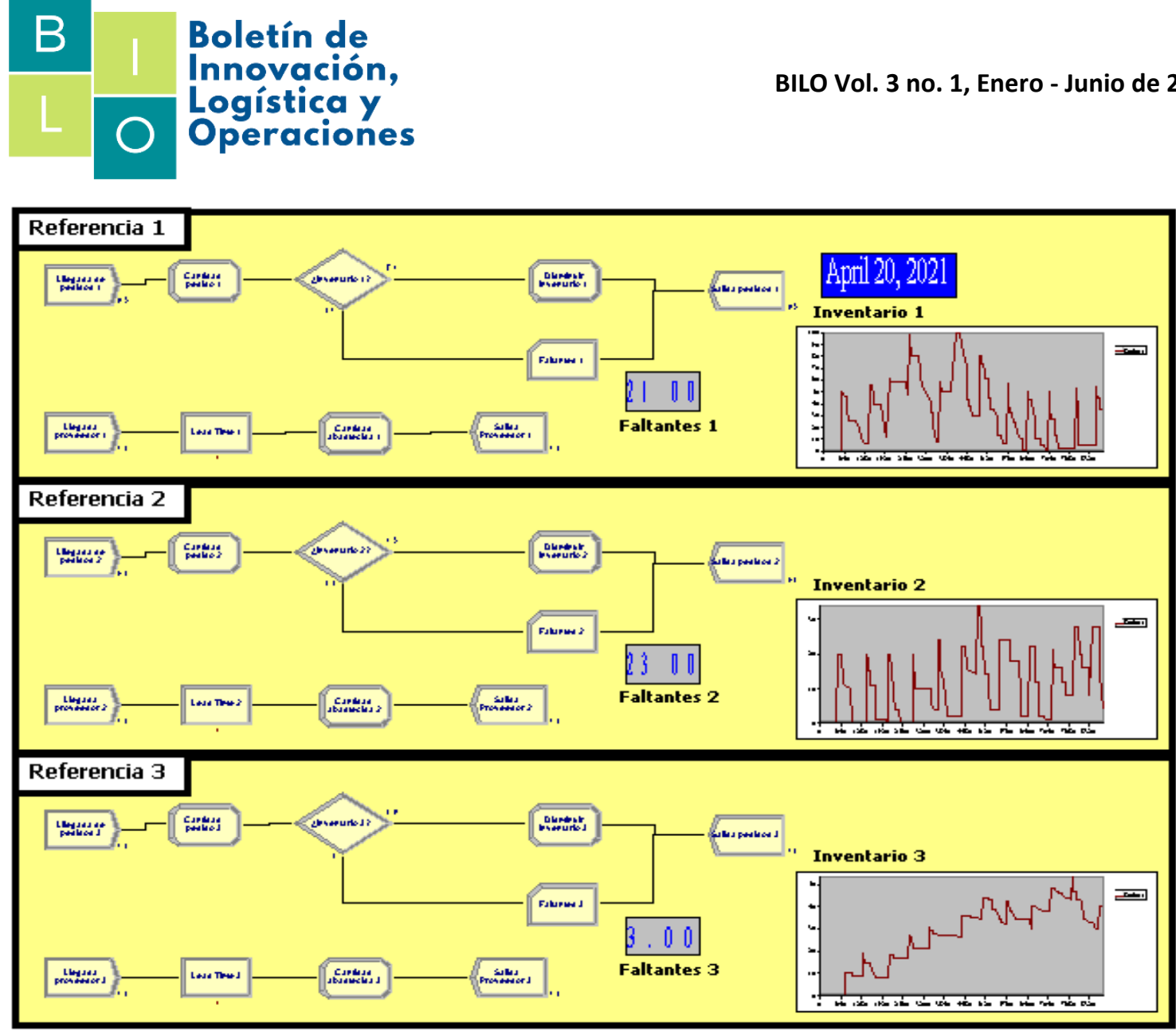

Figura 7. Modelo en el Software Arena.

El modelo fue validado teniendo en cuenta los siguientes datos de salida la cantidad de faltantes fue similar a lo encontrado con la prueba preliminar presentando errores por cada referencia de 13, 18 y $40 \%$ para cada referencia. Cabe resaltar que la gran diferencia presentada en la última referencia puede ser debida a los pocos faltantes que esta genera. Dado que el modelo refleja en gran medida lo que la realidad muestra, se empleara este modelo para estudiar diferentes propuestas de mejora.

\section{Análisis de salida}

A continuación, se realizará el análisis de salida del modelo propuesto, teniendo en cuenta como medida de desempeño la cantidad de faltante. Se establecerá una longitud de la corrida de un año, que es el horizonte de planeación definido por la compañía, y se determinaran el número de réplicas y el periodo de calentamiento. Para determinar el número de réplicas se utilizó el método de razón de ancho medio. Por consideración se desea que el valor de $\mathrm{h}$ no supere el $30 \%$ de lo obtenido para $\mathrm{h}_{0}$. Para esto la Tabla 3 resume los resultados encontrados.

Tabla 3. Número de réplicas.

\begin{tabular}{ccccccc}
\hline $\begin{array}{c}\text { Faltantes } \\
\text { referencia }\end{array}$ & Promedio & $\mathrm{h}_{0}$ & $\mathrm{n}_{0}$ & $\mathrm{~S}_{0}$ & $\mathrm{~h}$ & $\mathrm{n}$ \\
\hline 1 & 20 & 12,71 & 2 & 1,415 & 3,813 & 23 \\
2 & 22 & 12,71 & 2 & 1,415 & 3,813 & 23 \\
3 & 6,5 & 44,47 & 2 & 4,950 & 13,341 & 23 \\
\hline
\end{tabular}

Es decir, reduciendo un $30 \%$ la razón de ancho medio encontrada con la corrida preliminar, se tiene que el número de réplicas a utilizar será de 23. Para la estimación del periodo de calentamiento se utilizó el método gráfico, se graficaron los valores del tiempo entre faltantes promedios de producto 1 para este fin, se realizaron 6 réplicas y se tomaron datos para 30 días, la Figura 7 muestra lo obtenido. 

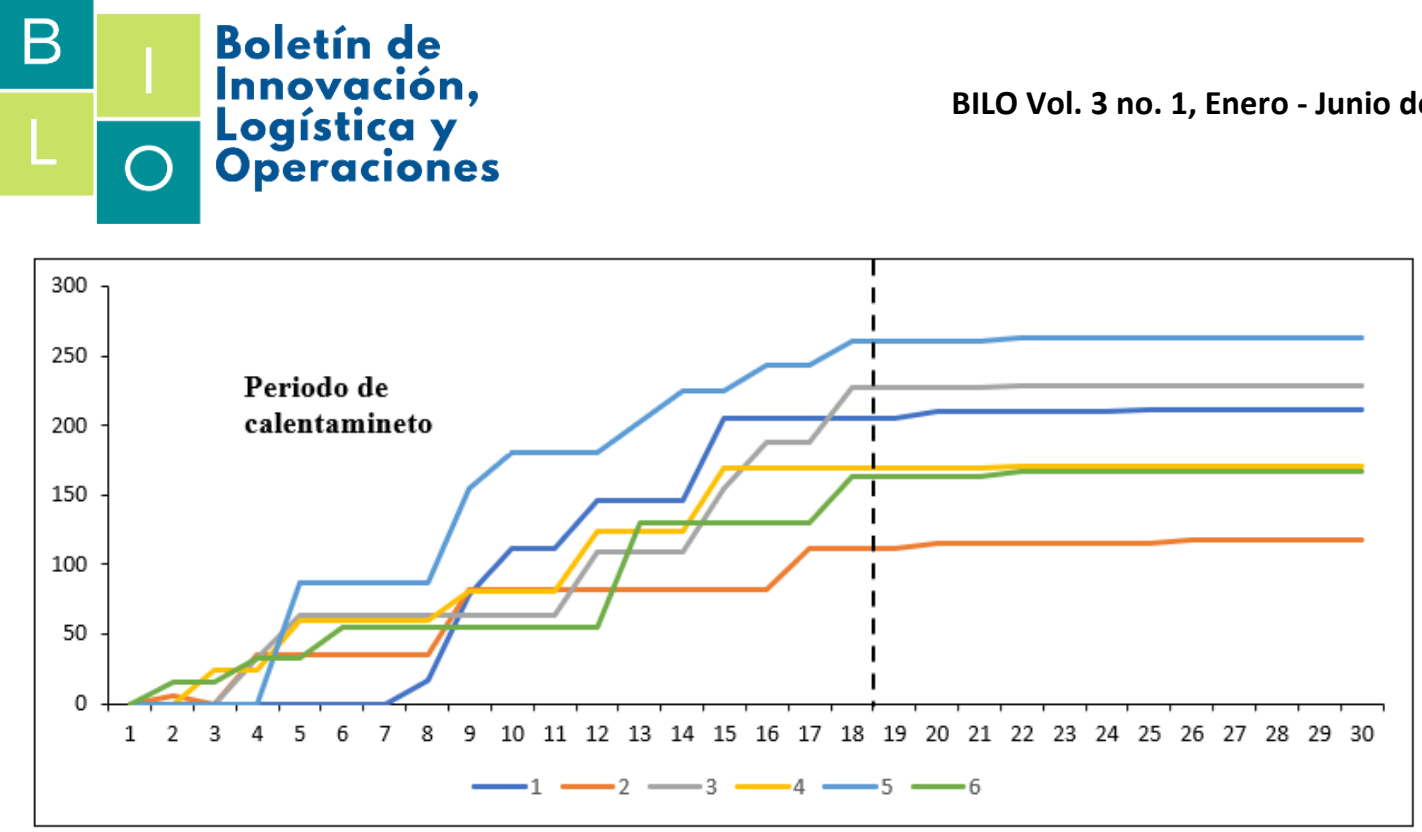

Figura 2. Periodo de calentamiento.

Como se muestra en la Figura 7, el periodo de calentamiento que deberá tomarse será de aproximadamente 18 días. Para darle tiempo a la simulación de que converjan sus resultados.

\section{Análisis de resultados}

Dado que la mayor parte de los faltantes presentados se encuentra en los primeros dos productos, las propuestas se realizarán en los mencionados sub sistemas para poder intervenir los productos 1 y 2 , estas propuestas se muestran en la Tabla 4.

Tabla 4. Propuestas de mejora.

\begin{tabular}{c|l}
\hline Propuesta & \multicolumn{1}{c}{ Descripción } \\
\hline 1 & $\begin{array}{l}\text { Aumentar la cantidad a pedir de producto } 1 \text { y } 2 \text { hasta } 60 \text { y } 30 \text { unidades, y } \\
\text { reducir la cantidad a pedir del producto } 3 \text { hasta } 8 .\end{array}$ \\
$\begin{array}{l}\text { Utilizar otro proveedor con tiempos de entrega menores (UNIF (10, 25)) con } \\
\text { lo cual se incurre en un sobrecosto de 5\%. } \\
\text { Utilizar otro proveedor con tiempos de entrega menores (UNIF (10, 25)) con } \\
\text { lo cual se incurre en un sobrecosto de 5\%., además de aumentar la cantidad a } \\
\text { pedir de producto } 1 \text { y } 2 \text { hasta } 55 \text { y } 35 \text { unidades., y reducir la cantidad a pedir } \\
\text { del producto } 3 \text { hasta } 6 .\end{array}$ \\
\hline
\end{tabular}

Las propuestas fueron probadas bajo las medidas encontradas en el análisis de salida, arrojando los siguientes resultados para las medidas de desempeño. Se tiene que considerar además que el costo de inventario es de aproximadamente 2.000 por unidad. Se calculo un costo promedio a partir del inventario de promedio de todos los productos luego de la simulación, esto como forma para decidir sobre una propuesta, ver Tabla 5.

Tabla 5. Análisis de escenarios

\begin{tabular}{cccccl}
\hline Propuesta & Falt 1 & Falt 2 & Falt 3 & Costo \\
\hline Actual & 19,6 & 22,2 & 1,2 & $\$ 136.555$ \\
1 & 7,6 & 9,4 & 1,4 & $\$ 133.534$ \\
2 & 17,4 & 21,4 & 0,2 & $\$ 140.087$ \\
3 & 6,2 & 6,4 & 2,2 & $\$ 140.119$ \\
\hline
\end{tabular}

\section{Conclusiones}

Como puede apreciarse en la Tabla 5 la mejor propuesta en cuanto a costo es la numero 1, la cual reduce el costo promedio de inventario en hasta \$ 133.534 reduciendo a la vez la cantidad de faltante drásticamente con respecto a la propuesta actual, sin embargo, la mejor propuesta en cuanto a faltante es la 3 con un sobrecosto presentado por la toma del nuevo proveedor; en base a la información limitada que se tiene sobre la empresa se aconseja emplear la propuesta 1 la cual no es muy invasiva ya que no afecta la relación cliente - proveedor, además de que no se están empleando más recursos solo es una decisión en basada en cuanto pedir. Como recomendación 


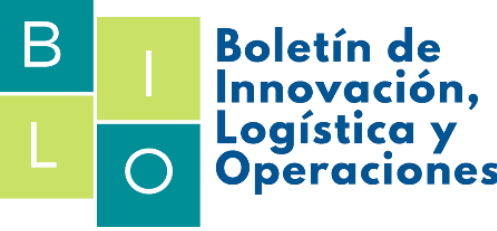

a la compañía, se deja a su criterio la puesta en marcha de alguna de las otras propuestas en cuanto a sus requerimientos especifico, y su relación con sus proveedores.

\section{Referencias}

[1] Patiño Builes, A. (2015). Tendencias tecnológicas que influyen en el aumento de la productividad empresarial. INGE CUC, 11(2), 84-96. https://doi.org/10.17981/ingecuc.11.2.2015.09.

[2] R. Jiraruttrakul, S. Smutkupt, W. Marksin, L. Liu, and C. Thanathawee, “APPLYING AN EOQ MODEL TO REDUCE AN INVENTORY COST,” J. Supply Chain Manag. Res. Pract., vol. 11, no. 1, pp. 46-55, 2017.

[3] J. L. C. Corea, J. E. D. Camejo, O. D. F. Espinoza, and H. Y. L. Gutiérrez, "POLÍTICA DE INVENTARIOS

[4] A. Mogrovejo Del Salto, "Estimar mediante simulación el inventario a comprar considerando predicciones de la demanda de los productos," Universidad Politécnica de Catalunya, 2018.

[5] N. Baharom and P. Hamzah, "Inventory Optimization Using Simulation Approach,” J. Comput. Res. Innov., vol. 3, no. 2, pp. 38-47, 2018.

[6] M. M. Molina Aguirre and A. G. Flórez Gómez, DISEÑO DE LA DISTRIBUCIÓN FÍSICA DE LA BODEGA DE MERCADEO DE LA UNIVERSIDAD DEL NORTE PARA MEJORAR LAS DISTANCIAS RECORRIDAS. Universidad del Norte, 2018.

[7] D. P. LOPEZ PALLARES and L. M. FONTALVO MERCADO, "DISEÑO DE UNA PROPUESTA DE MEJORA PARA LA REPOSICIÓN DE INVENTARIOS A TRAVES DE LA SIMULACION EN LA LINEA DE VEHICULOS LIVIANOS DE UNA EMPRESA DISTRIBUIDORA DE AUTOPARTES," UNIVERSIDAD DE LA COSTA, CUC, 2019.

[8] V. T. Kim Cuc, N. Truong Thi, and N. T. Le Thuy, "A Simulation Modelling Approach for Selection of Inventory Policy in a Supply Chain," in International Conference on System Science and Engineering (ICSSE), 2019, pp. 349-354.

[9] G. Xu, J. Feng, F. Chen, H. Wang, and Z. Wang, "Simulation-based optimization of control policy on multi-echelon inventory system for fresh agricultural products," Int. jourrnal Agric. Biol. Eng., vol. 12, no. 2, pp. 184-194, 2019, [Online]. Available: http://www.ijabe.org/index.php/ijabe/article/view/2834

[10] J. Reyes et al., "A Study on Modeling and Simulation of Automobile Painting Process Based on Flexsim," in International Conference on Computer Science, Electronics and Industrial Engineering (CSEI), 2019, pp. 260-273, [Online]. Available: https://link.springer.com/chapter/10.1007/978-3-03033614-1_18

[11] C. A. RESTREPO ZULUAGA and C. A. AGUDELO MURILLAS, DIAGNÓSTICO Y PROPUESTA PARA EL MEJORAMIENTO DEL SISTEMA DE GESTIÓN DE INVENTARIOS EN LA FERRETERIA Y DEPÓSITO LAS PALMAS S.A.S. 2016.

[12] S. A. Khan, F. Dweiri, and A. Chaabane, "MANAGING WAREHOUSE INVENTORY: A SIMULATION BASED CASE STUDY," in 11th International Conference on Modeling, Optimization and Simulation - MOSIM'16, 2016.

[13] M. A. Samad and V. Anand, "Inventory Simulation Model of a Lamp of Maintenance Warehouse of Facilities Management Department at Southeast Missouri State University Using Arena," Univers. J. Eng. Sci., vol. 4, no. 2, pp. 15-21, 2016.

[14] J. R. Cantú González, M. del C. Guardado García, and J. L. Balderas Herrera, "SIMULACIÓN DE PROCESOS, UNA PERSPECTIVA EN PRO DEL DESEMPEÑO OPERACIONAL,” Rev. Iberoam. Prod. Académica y Gestión Educ., vol. 3, no. 5, 2016.

[15] B. V. S. Suraj, S. S. Kumar, and S. Routroy, "Positioning of Inventory in Supply Chain Using 


\section{Operaciones}

Simulation Modeling," IUP J. Supply Chain Manag., vol. 13, no. 2, pp. 20-32, 2016.

[16] M. A. Álvarez Rangel, "Simulación de un sistema de surtido de rollos para el área de corte," 2017.

[17] R. D. Diuza Vallejo, "Diseño de una metodología para el uso de la simulación como herramienta para la enseñanza de la gestión de operaciones en la cadena de suministro," Universidad Autónoma del occidente, 2016.

[18] A. Parra Arenas, "Propuestas de mejora del sistema de distribución de aguacate hass para las exportaciones colombianas mediante simulación," Universidad autónoma de nuevo león, 2018.

[19] A. A. Giubergia, V. Gil Costa, Y. Mansilla, D. Narváez, M. E. Bertello, and M. Besso, "Simulación aplicada al cálculo de capacidades de almacenamiento y stock piles," Minería y Geol., vol. 32, no. 2, pp. 70-86, 2016, [Online]. Available: https://www.redalyc.org/pdf/2235/223545820005.pdf

[20] S. Huihui, M. Xiaoxia, and M. Xiangguo, "Simulation and Optimization of Warehouse Operation Based on Flexsim," J. Appl. Sci. Eng. Innov., vol. 3, no. 4, pp. 125-128, 2016, [Online]. Available: http://www.jasei.org/PDF/3-4/3-125-128.pdf

[21] D.C. Landinez-Lamadrid, D. G. Ramirez-Ríos, D. Neira Rodado, K. Parra Negrete and J.P. Combita Niño "Shapley Value: its algorithms and application to supply chains," INGE CUC, vol. 13, no. 1, 2017. DOI: http://dx.doi.org/10.17981/ingecuc.13.1.2017.06 